Supporting Information

\title{
Nanoparticle-Induced Ellipse-to-Vesicle Morphology Transition of Rod-Coil-Rod Triblock Copolymer Aggregates
}

\author{
Chaoying Yang, Qing Li, Chunhua Cai, ${ }^{*}$ and Jiaping Lin ${ }^{*}$
}

Shanghai Key Laboratory of Advanced Polymeric Materials, State Key Laboratory of Bioreactor

Engineering, Key Laboratory for Ultrafine Materials of Ministry of Education, School of Materials

Science and Engineering, East China University of Science and Technology, Shanghai 200237, China

* Corresponding author.

E-mail: jlin@ecust.edu.cn (J.L.), caichunhua@ecust.edu.cn (C.C.); Tel: +86-21-64253370 


\section{Synthesis of copolymers and gold nanoparticles}

Materials and reagents. $\mathrm{o}, \mathrm{o}^{\prime}-\mathrm{Bis}\left(2\right.$-aminoethyl)polyethylene glycol $\left(\mathrm{H}_{2} \mathrm{~N}-\mathrm{PEG}-\mathrm{NH}_{2}, M_{\mathrm{n}}=6000\right)$, gold(III) chloride trihydrate $\left(\mathrm{HAuCl}_{4} \cdot 3 \mathrm{H}_{2} \mathrm{O},>99.9 \%\right)$, and sodium borohydride $\left(\mathrm{NaBH}_{4}, \geq 98.0 \%\right)$ were purchased from Sigma-Aldrich. Oleylamine (OAm, C18 content: 80\%-90\%) was purchased from Acros Organics. Analytical grade of 1,4-dioxane was refluxed with sodium and distilled immediately before use. All the other reagents were purchased from Adamas-beta, and used without further purification or otherwise specified. $\gamma$-benzyl-L-glutamate- $N$-carboxyanhydride (BLG-NCA) was synthesized according to the procedures reported in the literature. ${ }^{\mathrm{S}-\mathrm{S} 3}$

Synthesis of PBLG-b-PEG-b-PBLG (BEB) triblock copolymers. BEB triblock copolymers were synthesized by ring-opening polymerization of BLG-NCA initiated by the terminal amino groups of $\mathrm{H}_{2} \mathrm{~N}-\mathrm{PEG}-\mathrm{NH}_{2}$ with anhydrous 1 ,4-dioxane as solvent. ${ }^{\mathrm{S} 1, \mathrm{~S} 2}$ The reactions were performed in a flame-dried reaction bottle under a dry nitrogen atmosphere at room temperature. After 3 days, the reaction mixture was poured into a large volume of anhydrous ethanol to precipitate the BEB triblock copolymer. The precipitated product was dried under vacuum and then purified twice by repeated precipitation from a chloroform solution into a large volume of anhydrous methanol. Finally, the product was dried under vacuum and white power was collected.

Synthesis of gold nanoparticles (AuNPs). Oleylamine-stabilized small AuNPs (AuOAm1) were prepared according to procedures reported in literatures. ${ }^{\mathrm{S4}, \mathrm{S} 5}$ Typical procedures employed are as follows. Tetralin $(40 \mathrm{~mL})$, oleylamine $(40 \mathrm{~mL})$, and $\mathrm{HAuCl}_{4} \cdot 3 \mathrm{H}_{2} \mathrm{O}(0.4 \mathrm{~g}, 1.02 \mathrm{mmol})$ were charged into a flask, and then magnetically stirred at $25{ }^{\circ} \mathrm{C}$ under $\mathrm{N}_{2}$ atmosphere. After 30 min, a reducing solution containing tert-butylamine-borane complex (TBAB) $(0.174 \mathrm{~g}, 2.0 \mathrm{mmol})$, tetralin $(4.0 \mathrm{~mL})$, and oleylamine $(4.0 \mathrm{~mL})$ was quickly injected into the precursor solution. After further reacting at $25{ }^{\circ} \mathrm{C}$ 
under $\mathrm{N}_{2}$ atmosphere for $1 \mathrm{~h}$, the obtained oleylamine-stabilized AuNPs was then precipitated into an excess of acetone $(200 \mathrm{~mL})$ and collected by centrifugation $(10000 \mathrm{rpm}, 10 \mathrm{~min})$, washed with acetone three times to remove excess oleylamine.

Oleylamine-stabilized large AuNPs (AuOAm2) were prepared using a different method with AuOAm1. ${ }^{\mathrm{S} 6}$ Briefly, a solution containing $0.25 \mathrm{~g}(0.64 \mathrm{mmol})$ of $\mathrm{HAuCl}_{4} \cdot 3 \mathrm{H}_{2} \mathrm{O}, 2.0 \mathrm{~g}(7.4 \mathrm{mmol}) \mathrm{of}$ oleylamine and $5.0 \mathrm{~mL}$ of toluene was quickly injected into $100 \mathrm{~mL}$ boiling toluene solution of $3.4 \mathrm{~g}$ (12.8 mmol) oleylamine. Reaction was stopped after 2 hours, and $200 \mathrm{~mL}$ of methanol were added to precipitate the product. The particles were isolated by centrifugation ( $8500 \mathrm{rpm}, 10 \mathrm{~min})$, and washed three times with $100 \mathrm{~mL}$ portions of methanol to remove unreacted oleylamine.

1-dodecanethiol-stabilized small AuNPs (AuDT) were synthesized by the Brust-Schiffrin method with slight modification. ${ }^{\mathrm{S} 7}$ Typically, $0.30 \mathrm{~g}(0.76 \mathrm{mmol})$ of $\mathrm{HAuCl}_{4} \cdot 3 \mathrm{H}_{2} \mathrm{O}$ was dissolved in $20.0 \mathrm{~mL}$ of water, and $1.0 \mathrm{~g}$ cetyltrimethyl ammonium bromide (CTAB) was dissolved in a mixture solvent containing $100 \mathrm{~mL}$ chloroform and $50 \mathrm{~mL}$ toluene, respectively. The two solutions were mixed and stirred until gold precursors were transferred to the organic phase and collected by separating funnel. To the organic phase, $0.4 \mathrm{~mL}$ of 1-dodecanethiol (DT, $1.67 \mathrm{mmol}$ ) was added and then the mixture was left stirring for $30 \mathrm{~min}$. The reducing agent, $\mathrm{NaBH}_{4}(8.0 \mathrm{mmol}$ in $10 \mathrm{~mL}$ water $)$ was slowly added to the mixture with vigorous stirring. After further stirring for $3.0 \mathrm{~h}$, the organic phase was separated and evaporated to $10 \mathrm{~mL}$ in a rotary evaporator. An excess amount of ethanol was added to precipitate the nanoparticles. The mixture was kept for $10 \mathrm{~h}$ at $-18^{\circ} \mathrm{C}$ and the dark brown precipitate was collected and dispersed in toluene. Finally, the nanoparticles were washed one more time with ethanol to remove excess 1-dodecanethiol. 


\section{Characterization of the PBLG- $b$-PEG- $b$-PBLG triblock copolymers}

The structure of the PBLG- $b$-PEG- $b$-PBLG (BEB) triblock copolymers was characterized by Circular Dichroism (CD), ${ }^{1} \mathrm{H}$ NMR, and GPC. The assignment of the polypeptide secondary structures can be confirmed by CD. As shown in Figure S1, the CD spectra of BEB triblock copolymers in THF revealed a negative peak around $222 \mathrm{~nm}$, which was characteristic of $\alpha$-helices. ${ }^{\mathrm{S} 8}$ Thus the BEB triblock copolymer can be employed as a model of rod-coil-rod polymeric system. Note that the negative peak of BEB434 shifts to higher wavelength. This phenomenon could be attributed to the slight aggregation of copolymer in THF due to poor solubility of BEB434 with high molecular weight.

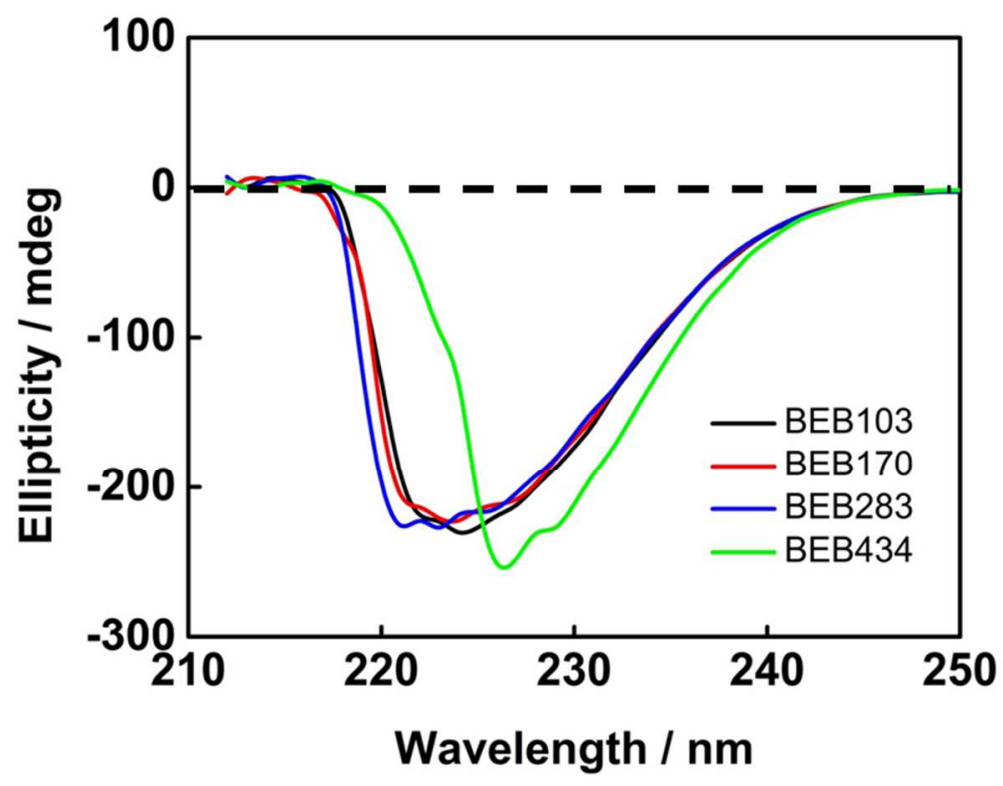

Figure S1. CD spectra of BEB103, BEB170, BEB283, and BEB434 triblock copolymers in THF. 
The molecular weight of the BEB triblock copolymer was calculated according to ${ }^{1} \mathrm{H}$ NMR spectrum (Figure S2a). Since the degree of polymerization (DP) of the PEG block is known (136), the molecular weight of PBLG block can be calculated by the peak intensities of the methylene proton signal (5.1 ppm) of PBLG and the methylene proton signal (3.6 ppm) of PEG. According to the NMR analysis, the DP and molecular weight of each PBLG block are 283 and 62000, respectively. GPC analysis in DMF reveals monomodal symmetric distribution with a relatively narrow PDI value of 1.25 (Figure S2b), which indicates a well-controlled polymerization process. More triblock copolymers with the same PEG block length but various PBLG block lengths were also synthesized, the detailed characterizations for these triblock copolymers were presented in Table S1.
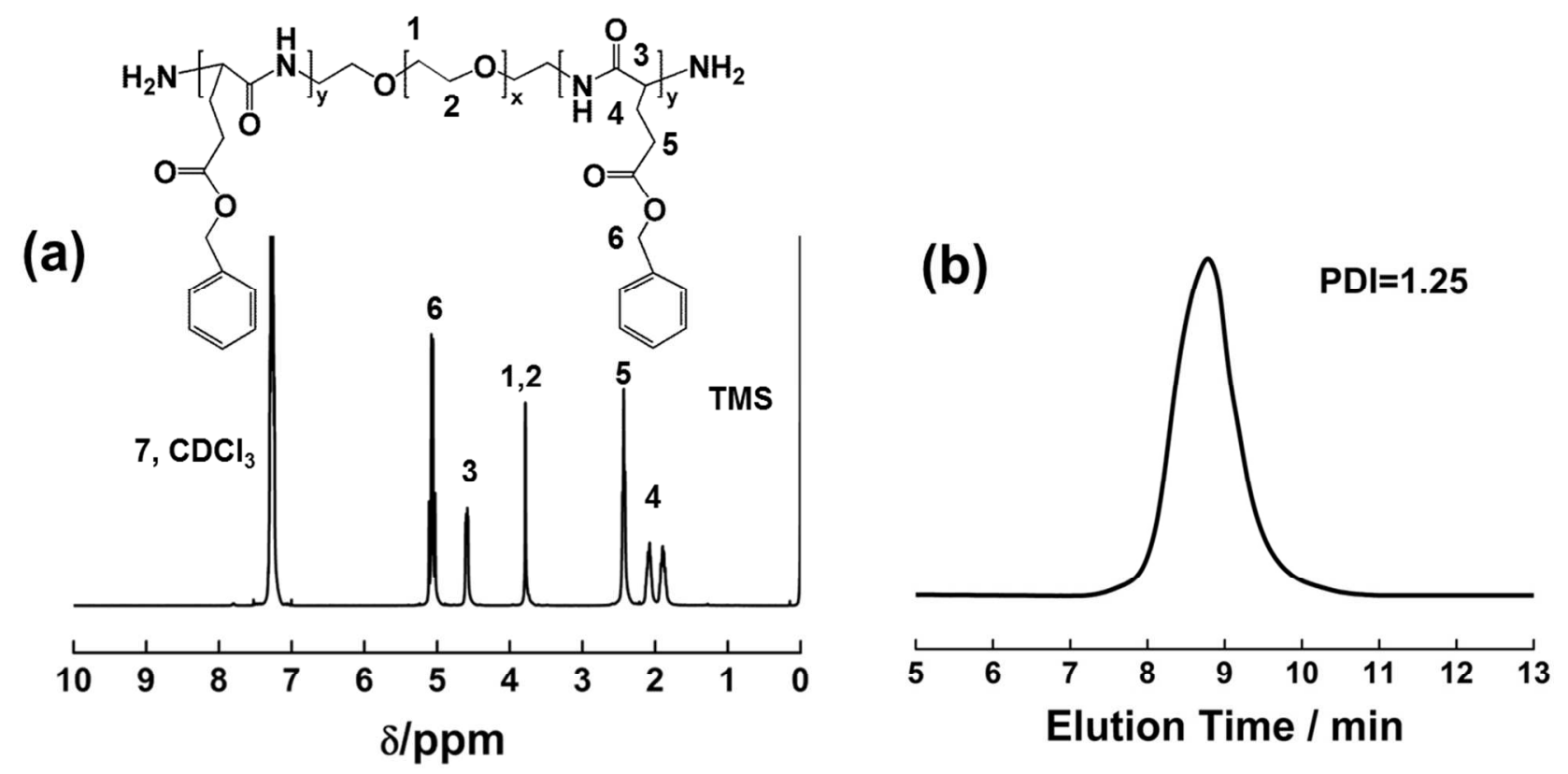

Figure S2. (a) ${ }^{1} \mathrm{H}$ NMR spectrum of the BEB283 triblock copolymer in $\mathrm{CDCl}_{3}$ with $15 \%$ TFA-d. (b) GPC trace of BEB283 triblock copolymer with DMF as eluent solvent and narrow polydispersity polystyrene as standards. 
Table S1. Characteristics of BEB triblock copolymers.

\begin{tabular}{|c|c|c|c|}
\hline Sample & $\mathrm{DP}_{\mathrm{PBLG}}^{\mathrm{a}}$ & $M_{\mathrm{n}}^{\mathrm{b}}$ & $M_{\mathrm{w}} / M_{\mathrm{n}}{ }^{\mathrm{c}}$ \\
\hline BEB103 & 103 & 51500 & 1.21 \\
\hline BEB170 & 170 & 80460 & 1.18 \\
\hline BEB283 & 283 & 131000 & 1.25 \\
\hline BEB434 & 434 & 196100 & 1.38 \\
\hline
\end{tabular}




\section{Size statistics of AuNPs}
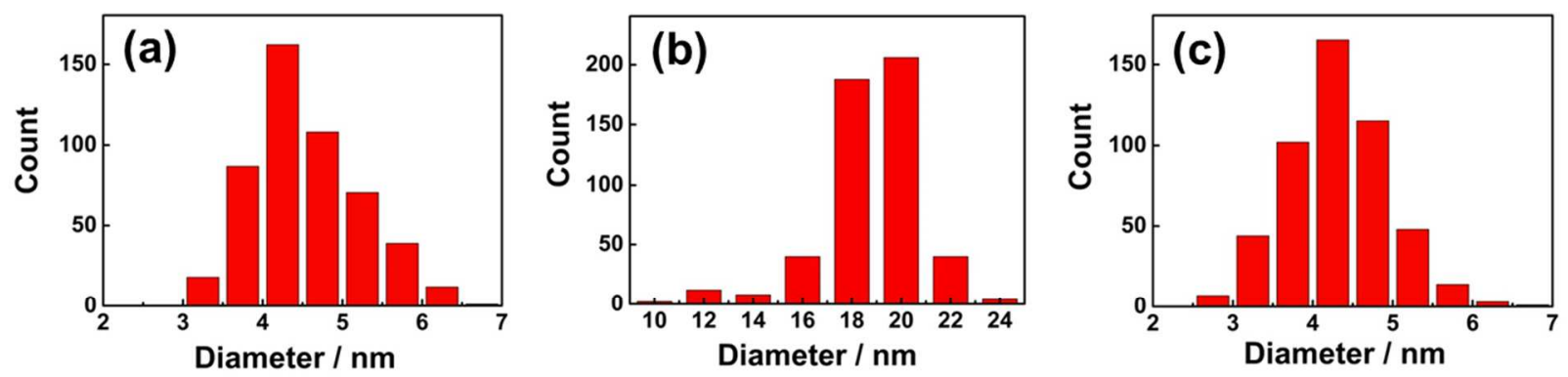

Figure S3. Size distributions of AuNPs. (a) AuOAm1, d = $4.5 \pm 0.9 \mathrm{~nm}$, (b) AuOAm2, d $=18.9 \pm 2.1$ $\mathrm{nm}$, and (c) AuDT, $\mathrm{d}=4.3 \pm 0.6 \mathrm{~nm}$. The size distribution of AuNPs was obtained by measuring 500 AuNPs from TEM images. 


\section{Effect of PBLG block length on the self-assembly behavior of BEB triblock}

\section{copolymer}

Generally, the volume fraction of the core-forming blocks should play an important role in the self-assembly of block copolymers (BCPs). For typical coil-coil block copolymer, when the volume fraction of the hydrophobic blocks increases, the sphere-to-vesicle morphology transition is preferred. Usually, when further increasing volume fraction of hydrophobic blocks, vesicles could be still obtained. In some cases, a vesicle-to-large-compound micelle morphology transition occurred.

Due to the rigidity nature and ordered packing tendency of the PBLG blocks, the BEB triblock copolymers exhibit distinct self-assembly behaviors. When BEB triblock copolymers contain longer PBLG rods, ellipse-like aggregates rather than vesicles are formed. When PBLG block length decreases, the vesicles were observed (Figure S4). Such an ellipse-to-vesicle morphology transition is attributed to the fact that the orientation order is reduced as rod blocks become shorter. According to our previous

work and the reports in literatures, ${ }^{\mathrm{S} 1, \mathrm{~S} 9, \mathrm{~S} 10}$ when PBLG rods are relatively longer, the interaction between PBLG rods increases. Therefore, the BEB triblock copolymers self-assemble into ellipse-like aggregates, in which the PBLG rods are in a favor form of parallel and dense arrangement. When PBLG rods are relatively shorter, the interaction between PBLG rods is weaker and the orientation order is reduced; as a result, the PBLG rods are aligned parallelly with each other to form the monolayer vesicle. Apparently, the ellipse-to-vesicle morphology transition can be attributed to change of the ordered and dense packing between PBLG rods as discussed above. 


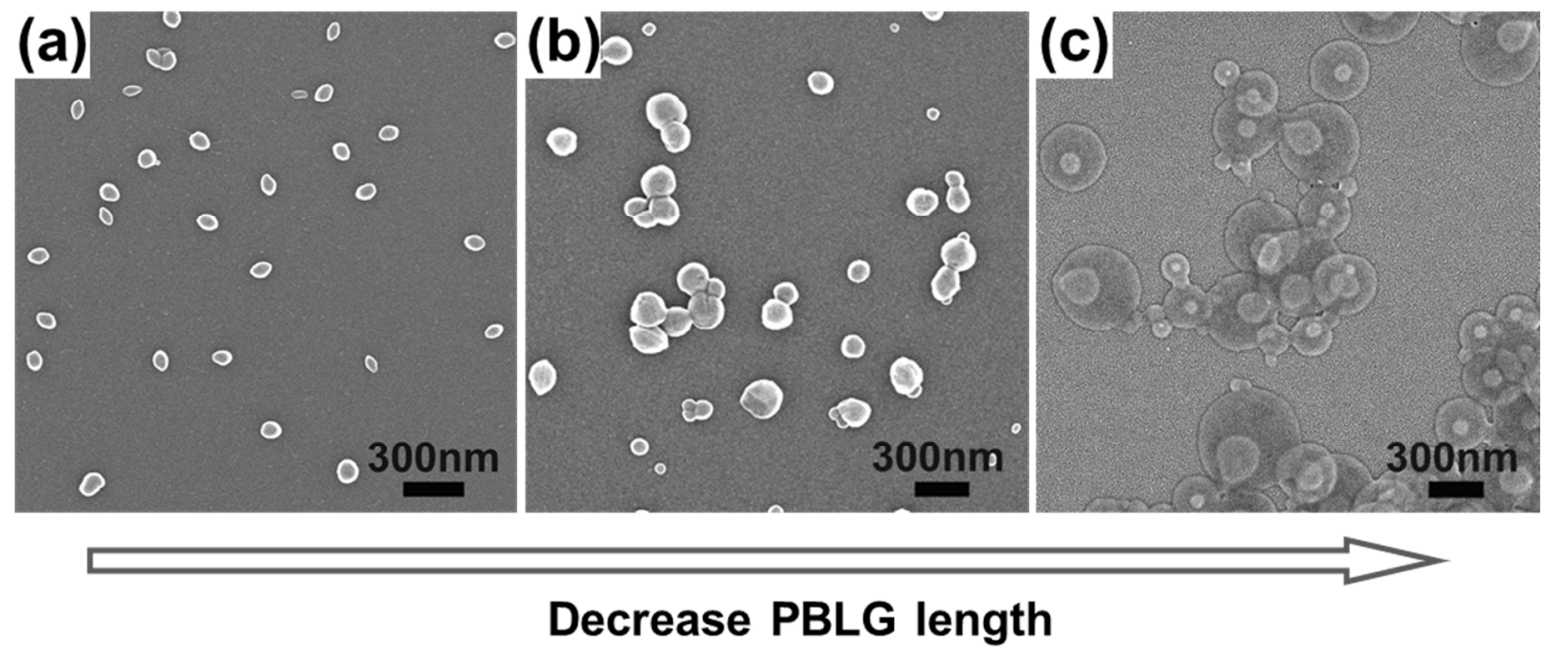

Figure S4. Typical SEM images of aggregates formed by triblock copolymers with various PBLG lengths: (a) BEB283, (b) BEB170, and (c) BEB103. 


\section{Cooperative self-assembly behavior of BEB triblock copolymer and AuNPs}

In order to study the effect of nanoparticle size on self-assembly behavior of BEB triblock copolymers, we synthesized medium-sized AuNPs according to the literature (larger than AuOAm1 but smaller than AuOAm2). Figure S5a and b show the TEM image and size histogram of the AuNPs coated with oleylamine (defined as AuOAm3), respectively. Size histogram reveals that the average diameter of AuOAm3 is $10.4 \mathrm{~nm}$. Cooperative self-assembly behavior of BEB triblock copolymers and AuOAm3 mixtures was investigated. As shown in Figure S5c and d, the vesicles were formed by $\mathrm{BEB} / \mathrm{AuOAm} 3$ mixtures at $f_{\mathrm{OAm} 3}=0.35$ and the nanoparticles were found to be uniformly distributed in the vesicle wall. The results indicated that the morphology transition from ellipse-to-vesicle can also be induced by AuNPs with medium size.

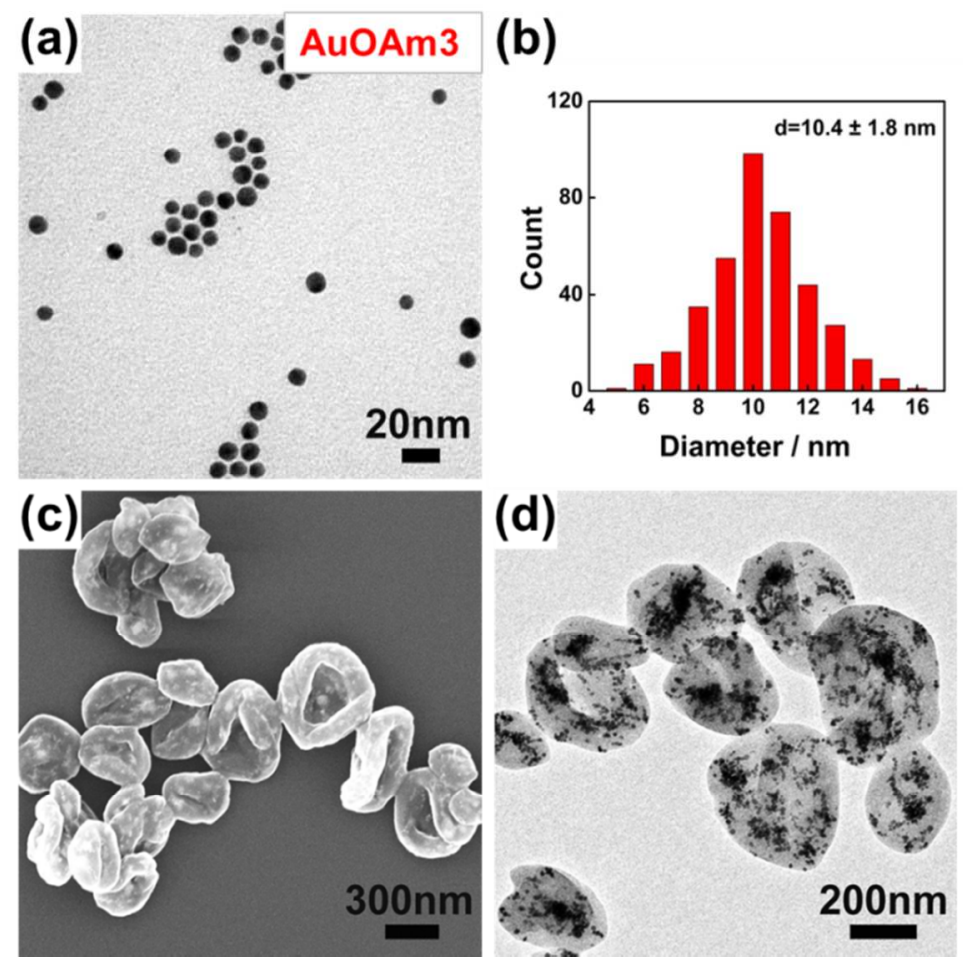

Figure S5. (a) TEM image and (b) size histogram of the AuNPs coated with oleylamine (defined as AuOAm3). $d=10.4 \pm 1.8 \mathrm{~nm}$. (c) SEM and (d) TEM images of the vesicles formed by BEB/AuOAm3 mixtures at $f_{\mathrm{OAm} 3}=0.35$. 
The effect of nanoparticles coated with 1-dodecanethiol (AuDT) on the self-assembly behavior of BEB triblock copolymer was investigated. Figure S6a-e show the SEM images of the aggregates formed at various $f_{\mathrm{AuDT}}$. As can be seen, the number and size of the vesicles increase with the $f_{\mathrm{AuDT}}$ increasing. Pure hybrid vesicles were obtained at a higher $f_{\mathrm{AuDT}}=0.30$. The vesicle structure was confirmed by the AFM analysis. A typical AFM image of vesicle structure formed at $f_{\mathrm{AuDT}}=0.30$ is presented in Figure S6f. From the height profile along the white line in the figure, we can see the collapsed center of the vesicle. In addition, the micelle is about $110 \mathrm{~nm}$ in center-height but more than $500 \mathrm{~nm}$ in width, further proving the hollow structure of vesicle.
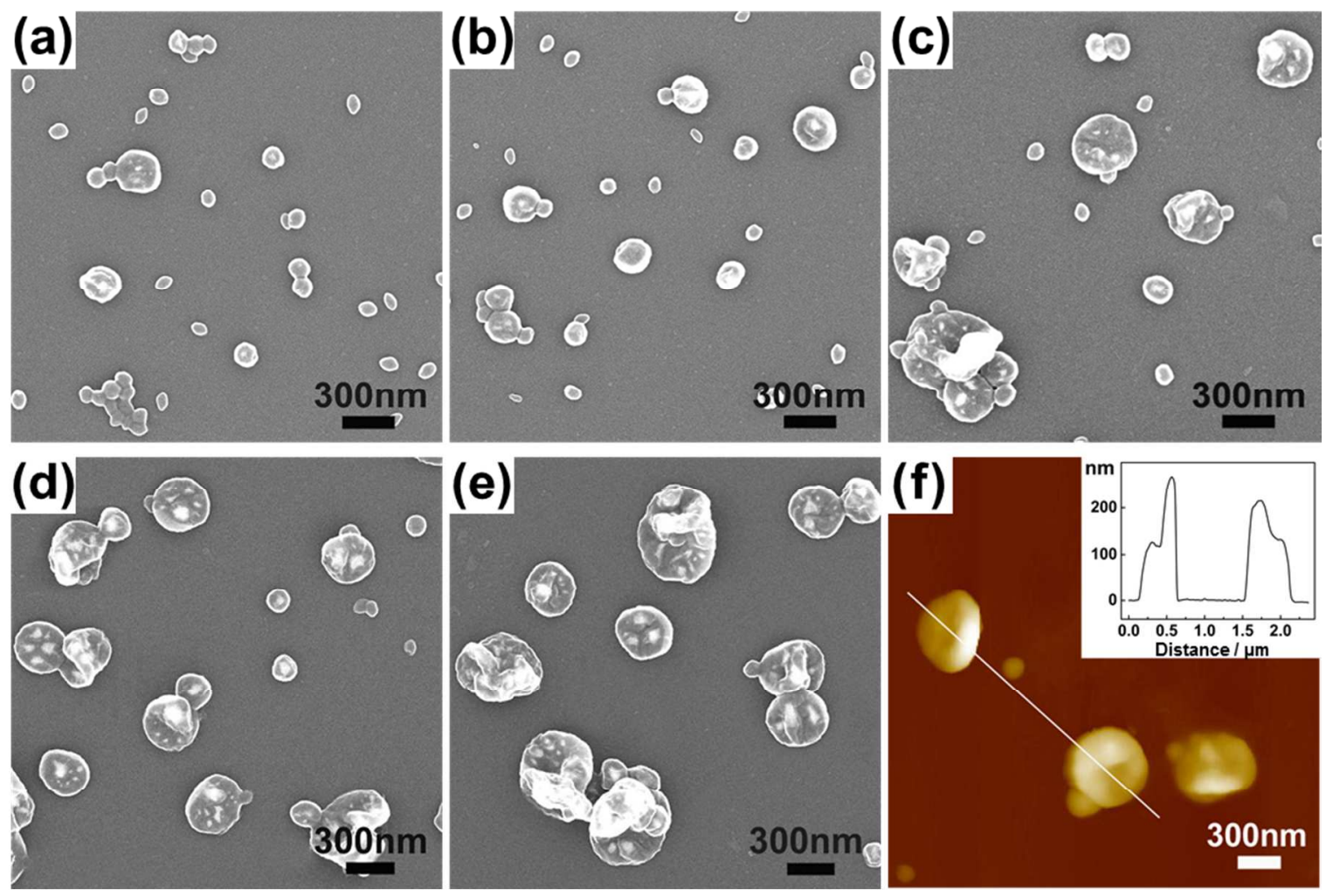

Figure S6. (a-e) SEM images of the aggregates formed by BEB/AuDT mixtures with various $f_{\mathrm{AuDT}}$ : (a) 0.05 , (b) 0.10 , (c) 0.17 , (d) 0.23 , and (e) 0.30. (f) AFM images of the vesicles formed by BEB/AuDT mixtures, $f_{\mathrm{AuDT}}=0.30$. Inset in (f) is the height profile along the white line in AFM image. 


\section{Simulation detalis}

\subsection{Simulation model of the rod-coil-rod triblock copolymer}

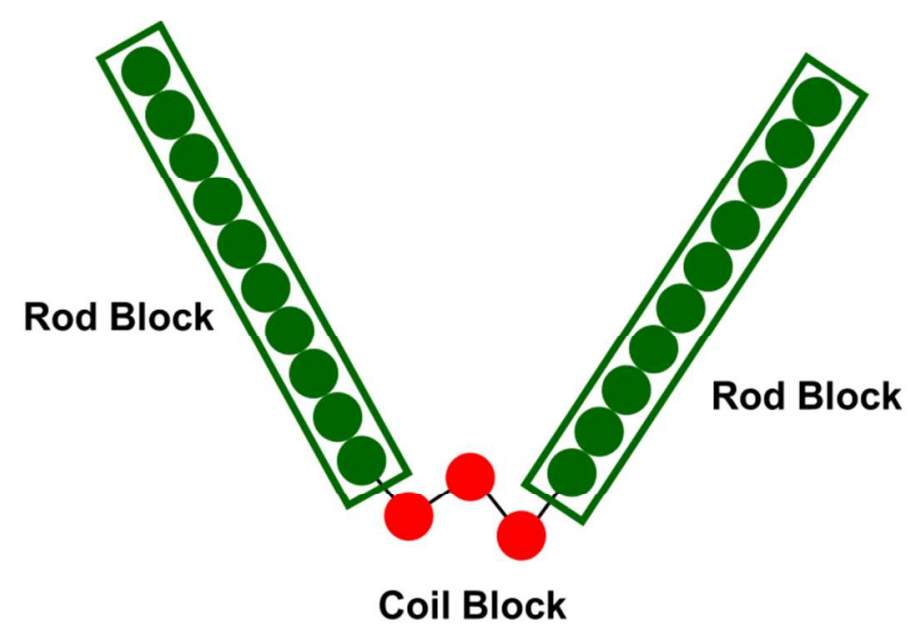

Figure S7. A DPD model of the rod-coil-rod triblock copolymer. The model consists of ten $\mathbf{R}$ beads on each rod block and three $\mathbf{C}$ beads on each coil block (denoted by $\mathbf{R}_{10} \mathbf{C}_{3} \mathbf{R}_{10}$ ). The DPD beads in rod blocks were linearly arranged and restricted to move as a rigid body. The distance between neighbor $\mathbf{R}$ beads was set as $0.86 r_{c}$, i.e. the equilibrium bond distance $r_{\mathrm{eq}}=0.86 r_{c}$. The coil block was modeled as a flexible polymeric chain.

The model triblock copolymer $\mathbf{R}_{10} \mathbf{C}_{3} \mathbf{R}_{10}$ constructed in our simulations corresponds to the $\mathrm{PBLG}_{283}-b-\mathrm{PEG}_{136}-b-\mathrm{PBLG}_{283}$ which was focused on in our experiments. The choice of the number of DPD beads in each rod block $\left(N_{\mathrm{R}}\right)$ and coil block $\left(N_{\mathrm{C}}\right)$ is based on our previous work and literatures. ${ }^{\mathrm{S} 11-\mathrm{S} 13}$ In the simulations, the mass of beads $(m=1)$ and the bead distance/equilibrium bond length $\left(0.86 r_{c}\right.$, where $r_{c}$ is the unit of length in simulations) for different beads were fixed. The molecular weights of rod blocks and coil blocks in $\mathrm{PBLG}_{283}-b-\mathrm{PEG}_{136}-b-\mathrm{PBLG}_{283}$ are 62000 and 6000 , respectively. First, the number of DPD beads was renormalized by keeping the bulk density identical in the simulations and experiments, and then a ratio of $62000 / 6000$ was obtained. In this case, 3.6 DPD 
beads for the PBLG form a $0.54 \mathrm{~nm}$ helix (or modeling rod), while $1 \mathrm{DPD}$ bead for the PEG occupies

$0.35 \mathrm{~nm} .{ }^{\mathrm{S} 12, \mathrm{~S} 13}$ Second, the number of DPD beads was renormalized by the length of rod blocks and coil blocks (the bead distance is equal to the equilibrium bond length), and we obtained the relative number of DPD beads for rod and coil blocks as $(62000 \times 0.54 / 3.6):(6000 \times 0.35 / 1) \approx 10: 2.26$. As a result, the model copolymer $\mathrm{R}_{10} \mathrm{C}_{3} \mathrm{R}_{10}$ was adopted in the present study.

The self-assembly behavior of model triblock copolymers with longer rod blocks, such as $\mathbf{R}_{12} \mathbf{C}_{3} \mathbf{R}_{12}$ and $\mathbf{R}_{14} \mathbf{C}_{3} \mathbf{R}_{14}$ were also studied in our simulations. It was found that the $\mathbf{R}_{12} \mathbf{C}_{3} \mathbf{R}_{12}$ also self-assembled into ellipse-like aggregates, and few coil blocks were distributed at the centers of ellipses. When nanoparticles were introduced, the vesicles were obtained. However, the $\mathbf{R}_{14} \mathbf{C}_{3} \mathbf{R}_{14}$ with even longer rods self-assembled into irregular aggregates. In addition, we didn't observe coil blocks at the centers of the aggregates. These structures are different from the ellipse-like structures formed by $\mathbf{R}_{10} \mathbf{C}_{3} \mathbf{R}_{10}$ (see Figure 2).

\subsection{Choice of the interaction parameter $a_{i j}$}

A table of interaction parameters $a_{i j}$ was provided in the main text (Table 1). Moreover, the choice of $a_{i j}$ is explained in details.

a) Interaction parameters $a_{i i}$ between DPD beads of the same species are set to be 25. This $a_{i i}$ is equal to the value calculated by Groot and Warren based on the compressibility of water. ${ }^{\mathrm{S} 14}$

b) Interaction parameters $a_{i j}$ between compatible species $\left(e . g ., a_{\mathrm{CS}}\right)$ are set to be 25 , whereas $a_{i j}$ between immiscible components are set to be larger than 25. The larger values of $a_{i j}$ between immiscible components correspond to the unfavorable interactions between them. ${ }^{\text {S15 }}$ 
c) The interaction parameters between rods and solvents $\left(a_{\mathrm{RS}}\right)$, between rods and coils $\left(a_{\mathrm{RC}}\right)$ and between particles and solvents $\left(a_{\mathrm{PS}}\right)$ were varied in the range from 30 to 75 when we simulated the morphological transition as shown in Figure $5\left(a_{\mathrm{RS}}=a_{\mathrm{RC}}=a_{\mathrm{PS}}\right)$. This stepwise change corresponds to the progressive addition of water in the experiment. ${ }^{\mathrm{S} 16, \mathrm{~S} 17}$

d) The interaction parameters between particles and solvents $\left(a_{\mathrm{PS}}\right)$, between particles and rods $\left(a_{\mathrm{PR}}\right)$ and between particles and coils $\left(a_{\mathrm{PC}}\right)$ were varied when we studied the hybrid vesicles containing different types of nanoparticles (Figure 8). We set $a_{\mathrm{PS}}=a_{\mathrm{PC}}=75$, and $a_{\mathrm{PR}}=25$ for AuOAm1 and AuOAm2, since we consider that the hydrophobicity of these nanoparticles is close to that of rod blocks. We set $a_{\mathrm{PS}}=a_{\mathrm{PC}}=200$, and $a_{\mathrm{PR}}=75$ for AuDT, because they are more hydrophobic than rod blocks and AuOAm1/AuOAm2. ${ }^{\text {S16,S17 }}$ 


\section{References}

(S1) Zhuang, Z.; Cai, C.; Jiang, T.; Lin, J.; Yang, C. Self-assembly behavior of rod-coil-rod polypeptide block copolymers. Polymer 2014, 55, 602-610.

(S2) Cai, C.; Zhang, L.; Lin, J.; Wang, L. Self-assembly behavior of pH- and thermosensitive amphiphilic triblock copolymers in solution: Experimental studies and self-consistent field theory simulations. J. Phys. Chem. B 2008, 112, 12666-12673.

(S3) Blout, E. R.; Karlson, R. H. Polypeptides. III. The synthesis of high molecular weight poly- $\gamma$-benzyl-L-glutamates1. J. Am. Chem. Soc. 1956, 78, 941-946.

(S4) Peng, S.; Lee, Y.; Wang, C.; Yin, H.; Dai, S.; Sun, S. A facile synthesis of monodisperse Au nanoparticles and their catalysis of CO oxidation. Nano Res. 2008, 1, 229-234.

(S5) Ku, K. H.; Shin, J. M.; Kim, M. P.; Lee, C.-H.; Seo, M.-K.; Yi, G.-R.; Jang, S. G.; Kim, B. J. Size-controlled nanoparticle-guided assembly of block copolymers for convex lens-shaped particles. $J$. Am. Chem. Soc. 2014, 136, 9982-9989.

(S6) Hiramatsu, H.; Osterloh, F. E. A simple large-scale synthesis of nearly monodisperse gold and silver nanoparticles with adjustable sizes and with exchangeable surfactants. Chem. Mater. 2004, 16, 2509-2511.

(S7) Brust, M.; Walker, M.; Bethell, D.; Schiffrin, D. J.; Whyman, R. Synthesis of thiol-derivatised gold nanoparticles in a two-phase liquid-liquid system. J. Chem. Soc., Chem. Commun. 1994, 801-802.

(S8) Cai, C.; Lin, J.; Chen, T.; Tian, X. Aggregation behavior of graft copolymer with rigid backbone. Langmuir 2010, 26, 2791-2797.

(S9) Lin, Y.-L.; Chang, H.-Y.; Sheng, Y.-J.; Tsao, H.-K. Structural and mechanical properties of polymersomes formed by rod-coil diblock copolymers. Soft Matter 2013, 9, 4802-4814. 
(S10) Lin, Y.-L.; Chang, H.-Y.; Sheng, Y.-J.; Tsao, H.-K. Self-assembled polymersomes formed by symmetric, asymmetric and side-chain-tethered coil-rod-coil triblock copolymers. Soft Matter 2014, 10, 1840-1852.

(S11) Cai, C.; Wang, L.; Lin, J.; Zhang, X. Morphology transformation of hybrid micelles self-assembled from rod-coil block copolymer and nanoparticles. Langmuir 2012, 28, 4515-4524.

(S12) Ortiz, V.; Nielsen, S. O.; Discher, D. E.; Klein, M. L.; Lipowsky, R.; Shillcock, J. Dissipative particle dynamics simulations of polymersomes. J. Phys. Chem. B 2005, 109, 17708-17714.

(S13) Ding, W.; Lin, S.; Lin, J.; Zhang, L. Effect of chain conformational change on micelle structures: Experimental studies and molecular dynamics simulations. J. Phys. Chem. B 2008, 112, 776-783.

(S14) Groot, R. D.; Warren, P. B. Dissipative particle dynamics: Bridging the gap between atomistic and mesoscopic simulation. J. Chem. Phys. 1997, 107, 4423-4435.

(S15) Dutt, M.; Kuksenok, O.; Nayhouse, M. J.; Little, S. R.; Balazs, A. C. Modeling the self-assembly of lipids and nanotubes in solution: Forming vesicles and bicelles with transmembrane nanotube channels. ACS Nano 2011, 5, 4769-4782.

(S16) Jiang, T.; Wang, L.; Lin, S.; Lin, J.; Li, Y. Structural evolution of multicompartment micelles self-assembled from linear ABC triblock copolymer in selective solvents. Langmuir 2011, 27, $6440-6448$.

(S17) Zhang, X.; Wang, L.; Zhang, L.; Lin, J.; Jiang, T. Controllable hierarchical microstructures self-assembled from multiblock copolymers confined in thin films. Langmuir 2015, 31, 2533-2544. 